\title{
Periodicity of Cycle Time in a U-Shaped Production Line with Heterogeneous Workers under Carousel Allocation
}

\author{
Mikihiko Hiraiwa, Koichi Nakade
}

Department of Architecture, Civil Engineering and Industrial Management Engineering, Nagoya Institute of Technology, Japan. Email: 16518507@stn.nitech.ac.jp,nakade@nitech.ac.jp

Received September 3, 2009; revised October 12, 2009; accepted November 17, 2009.

\begin{abstract}
A U-shaped production line with multiple machines and multiple workers is considered under carousel allocation in which all workers take charge of all machines in the same order. Nakade and Ohno (2003) show that, when the processing, operation and walking times are constant, the overall cycle time, which is a time interval between successive outputs of finished goods, is the greatest value of the maximal sum of the processing and operation times among machines and the time required for a worker to operate and walk around the production line without waiting for processing divided by the number of workers. In this paper, it is considered that operation times at each machine may be different between workers. If processing time is short, it is expected that the overall cycle time will be equal to the time for a worker to operate and walk around the line divided by the number of workers. However, under some specified cases, the overall cycle time is longer than that of this time, and the overall cycle time changes periodically. From numerical examples, it is shown that the order of arrivals of workers at machine 1 affects the overall cycle time. We give some properties on the periodicity of cycle times and discuss about cycle times.
\end{abstract}

Keywords: U-Shaped Production Line, Carousel Allocation, Operation Times, Cycle Time, Periodicity of Cycle Times

\section{Introduction}

In these days, U-shaped layout is commonly used in many production lines. In a U-shaped production line, there are two types of worker allocations, which are separate type and carousel type. In the separate allocation, each worker deals with a unique set of machines. In the carousel allocation, workers deal with all machines in the same route. In this paper, the carousel allocation is considered. An advantage of U-shaped layout is that it is easy to adjust the throughput of finished products from the last machine for fluctuation of demand by changing the number of workers [1,2].

Nakade and Ohno [3] have considered a U-shaped production line with multiple multi-function workers, and show the overall cycle time which is a time interval between successive outputs of finished products. When the processing, operation, and walking times are constant, the overall cycle time is the greatest value of the maximal sum of the processing and operation times among machines and the time required for a worker to operate and walk around the production line without waiting for processing divided by the number of workers. They assume that operation times at each machine are the same among workers.

Recently, many temp staffs are employed as workers and they are committed into production lines because of cost reduction. For example, many foreigners and part time workers are employed for a short span. Therefore it becomes difficult to maintain the workers well-skilled in the long time, so the worker's skills remain mutually different.

Nakade and Nishiwaki [4] have considered a Ushaped production line with multiple heterogeneous multi-function workers and propose an algorithm for computing an optimal allocation of workers to machines which minimizes the overall cycle time.

The formula of cycle time derived in Nakade and Nishiwaki [4] is simple and understandable, where it is assumed that different workers are assigned to the different machines. In the other allocation scheme, all workers have operations at all machines and as a carousel workers go around machines. We say this scheme carousel allocation. 
In this paper we consider the case that operation times at each machine are different among workers under carousel allocation. In this case, if the processing times are very small, then it may be guessed that the overall cycle time is equal to the greatest value of times required for each worker to operate and walk around the line divided by the number of workers. However, under some specified cases, the overall cycle time is larger than this time, and the overall cycle time changes periodically. We discuss the periodicity of cycle times and observe some properties on cycle times.

In the next section we describe a U-shaped production line. In Section 3 we show that the overall cycle time changes periodically by numerical examples and discuss the periodicity of cycle times. In Section 4 we conclude and discuss future research.

\section{A U-Shaped Production Line}

We consider a U-shaped production line with $K$ machines and $J$ workers, which is shown in Figure 1. It is assumed that $J \leq K$. There is enough raw material in front of machine 1 . The material is processed at machines 1 to $K$ sequentially, and customers receives finished products from machine $K$. Workers deal with all machines in the same route and operate an item at each machine. Operation times at each machine may be different among workers, and the operation time of worker $j$ at machine $k$ is denoted by $s_{j, k}$, which is deterministic. Worker $j$ starts his first operation at machine $k_{j}$ and visits machines $k_{j}+1, k_{j}+2, \cdots, K$, $1,2, \cdots$ sequentially. The deterministic walking time from machine $k$ to $k+1 \quad(k=1,2, \cdots, K)$ is denoted by $r_{k, k+1}$, where it is assumed that $K+1=1$. The processing time at machine $k$ is denoted by $i_{k}$, which is deterministic. When a worker visits a machine, if the preceding worker is operating at the machine or the

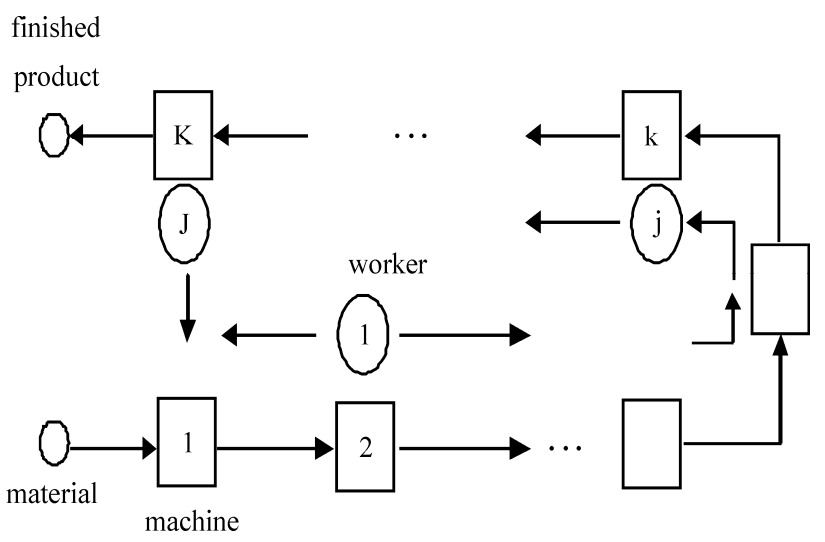

Figure 1. U-shaped production line machine is processing the preceding item, then the worker must wait for the completion of operating or processing. The overall cycle time is defined as a time interval between successive outputs of finished products. We also define the cycle time of worker $j$ as the time interval between the successive arrivals of worker $j$ at machine 1 . It is noted that the overall cycle time is the cycle time divided by the number of workers. The first cycle time of worker $j$ is the time interval between a start of first operation and the first arrival of worker $j$ at machine 1 . The $n$th $(n \geq 2)$ cycle time of worker $j$ is the time interval between the $(n-1)$ th and $n$th arrivals for worker $j$ at machine 1 .

\section{Cycle Time}

\subsection{Case of the Same Operation Times among Workers}

In the case that operating times at each machine are the same among workers, from Nakade and Ohno (2003), when processing, operation and walking times are constant, the overall cycle time $C^{\text {overall }}$ is expressed as

$$
C_{c}^{\text {overall }}=\max \left\{\max _{k \in K}\left(s_{k}+i_{k}\right), \frac{1}{J}\left(\sum_{k \in \hat{K}} s_{k}+\sum_{k \in \hat{K}} r_{k, k+1}\right)\right\} \text {, }
$$

where $s_{k}$ denotes the operation time at machine $k$ and $\hat{K}=\{1,2, \cdots, K\}$. The overall cycle time is the greatest value of the maximal sum of the processing and operation times among machines and the time required for a worker to operate and walk around the production line without waiting for processing divided by the number of workers.

\subsection{Case of Different Operation Times among Workers}

We consider the case that operating times at each machine may be different among workers. In this case, from Equation (1), the overall cycle time $C^{\text {overall }}$ may be guessed as

$$
\begin{array}{r}
C^{\text {overall }}=\max \left\{\max _{k \in \hat{K}}\left(\frac{1}{J} \sum_{j \in\{1,2, \cdots, J\}} s_{j, k}+i_{k}\right),\right. \\
\left.\frac{1}{J} \max _{j \in\{1,2, \cdots, J\}}\left(\sum_{k \in \hat{K}} s_{j, k}+\sum_{k \in \hat{K}} r_{k, k+1}\right)\right\} .
\end{array}
$$

In what follows, we consider the cycle time instead of the overall cycle time. Since the overall cycle time is the cycle time divided by the number of workers, the cycle time $C$ may be guessed as 


$$
\begin{aligned}
C=\max \left\{\max _{k \in \hat{K}}\left(\sum_{j \in\{1,2, \cdots, J\}} s_{j, k}+J i_{k}\right),\right. \\
\left.\max _{j \in\{1,2, \cdots, J\}}\left(\sum_{k \in \hat{K}} s_{j, k}+\sum_{k \in \hat{K}} r_{k, k+1}\right)\right\} .
\end{aligned}
$$

From Equation (3), when processing times are zero, it is guessed that the cycle time is equal to the greatest value among times required for each worker to operate and walk around the line. However, under some specified cases, the cycle time is greater than this value, and the cycle time changes periodically. The examples are shown in the next section.

\section{Periodicity of Cycle time}

\subsection{Numerical Examples}

The set of operation times of each worker at each machine is denoted by

$$
S_{l}=\left(\begin{array}{l}
s_{1,1}, \cdots, s_{1, K} \\
\vdots \cdots \cdots \cdots \\
s_{J, 1}, \cdots, s_{J, K}
\end{array}\right)
$$

where subscript $l$ is a number which distinguishes sets of operation times.

Let $J=3$ and $K=4$. It is assumed that processing times and walking times are zero, that is, $i_{k}=0$ and $r_{k, k+1}=0$ for $k \in \hat{K}$. Worker 1 starts his first operation at machine 1 , worker 2 at machine 2 and worker 3 at machine 3 . The first cycle is until the first arrival of a worker at machine 1 . The $n$th $(n \geq 2)$ cycle is the

Table 1. Cycle times for $S_{1}$

\begin{tabular}{cccc}
\hline cyclelworker & 1 & 2 & 3 \\
\hline 1 & 8.0 & 7.0 & 6.0 \\
2 & 8.0 & 8.0 & 8.0 \\
3 & 8.0 & 8.0 & 8.0 \\
4 & 8.0 & 8.0 & 8.0 \\
5 & 8.0 & 8.0 & 8.0 \\
6 & 8.0 & 8.0 & 8.0 \\
\hline
\end{tabular}

Table 2. Cycle times for $S_{2}$

\begin{tabular}{cccc}
\hline cyclelworker & 1 & 2 & 3 \\
\hline 1 & 8.0 & 7.0 & 2.0 \\
2 & 11.0 & 11.0 & 11.0 \\
3 & 8.0 & 8.0 & 8.0 \\
4 & 11.0 & 11.0 & 11.0 \\
5 & 8.0 & 8.0 & 8.0 \\
6 & 11.0 & 11.0 & 11.0 \\
\hline
\end{tabular}

interval between $(n-1)$ th and $n$th arrivals of a worker at machine 1 .

Cycle times, when operation times of each worker at each machine are $S_{1}=\left(\begin{array}{l}5,1,1,1 \\ 1,5,1,1 \\ 1,1,5,1\end{array}\right)$, are shown in Table 1 .

Cycle times of all workers are 8 at all cycles except for the first cycle. This is the case that the cycle time does not change periodically. Then the cycle time 8 in Table 1 is equal to the value which is derived from Equation (3).

Cycle times, when operation times of each worker at each machine are $S_{2}=\left(\begin{array}{l}5,1,1,1 \\ 1,1,5,1 \\ 1,5,1,1\end{array}\right)$, are shown in Table 2 . In this case, the cycle time changes periodically. Except for the first cycle, cycle times of each worker take values of 8 and 11 alternately. The cycle time 8 in Table 2 is equal to the value which is derived from Equation (3). The cycle time 11 is more than the value which is derived from Equation (3).

\subsection{Discussion}

We investigate many examples including the above, and we have the following properties on the cycle time.

1) In many cases, in which the difference is not so large among workers, the cycle time is the same as Equation (3).

2) If the cycle time changes periodically, then the period of the cycle time is two.

3) If the cycle time changes periodically, then the smaller cycle time is equal to the one which is derived from Equation (3), and the greater cycle time is more than the one which is derived from Equation (3).

4) Initial machines of each worker do not affect the cycle time if an order of arrivals of workers at machine 1 does not change, where, for example, the orders " $4,3,2,1$ " and " $2,1,4,3$ " are regarded as the same order.

5) An order of arrivals of workers at machine 1 affects the cycle time.

The movements of each worker when operation times are equal to $S_{2}$ are shown in Figure 2. When operation times are equal to $S_{2}$, for example, worker 2 tends to wait for the completion of operation of worker 3 at machine 2 since machine 2 is bottleneck for worker 3 . And also, since machine 3 is bottleneck for worker 2, worker 2 operates at his bottleneck machine (machine 3) after the waiting at machine 2. This makes the cycle time of worker 2 amplified. If a worker has his bottleneck machine after the bottleneck machine of his preceding worker, then the cycle time of the worker is amplified. In the next cycle, worker 3 finished his operation at his bottleneck machine (machine 2) while worker 2 operates at 


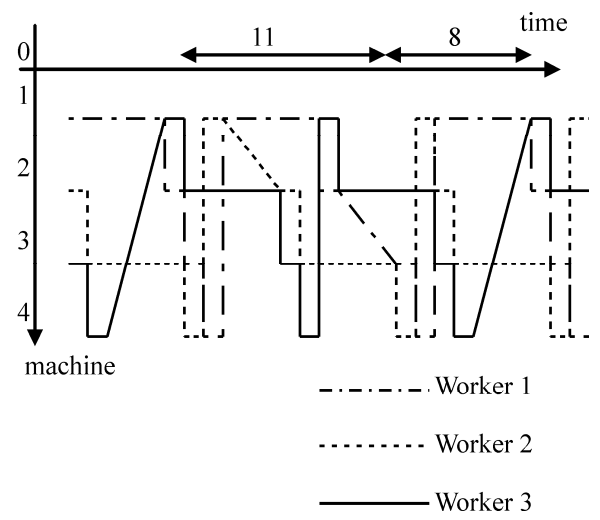

Figure 2. Movements of workers

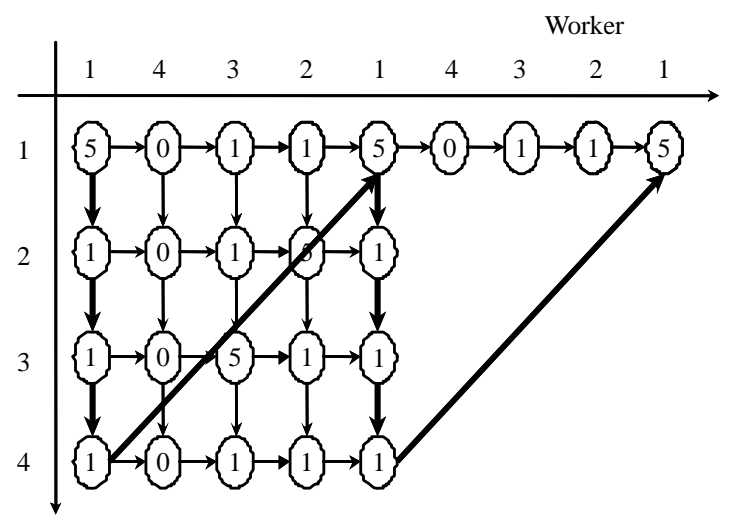

Figure 3. Arrow diagram for $S_{1}$

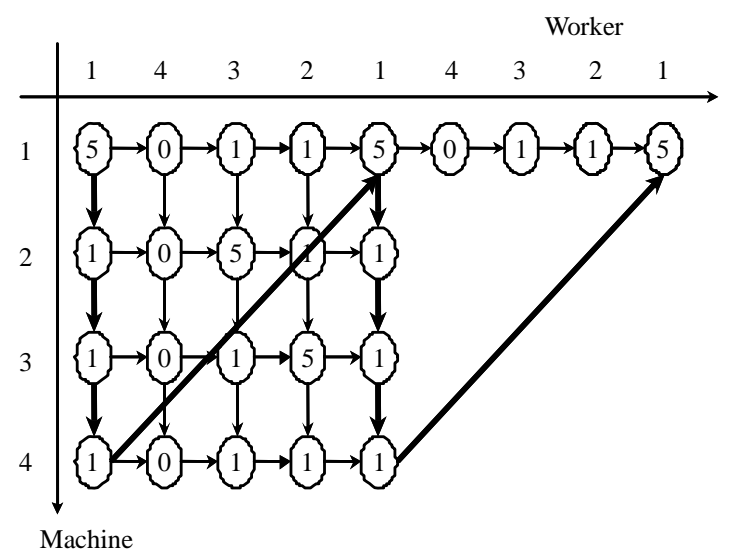

Figure 4. Arrow diagram for $S_{2}$

his bottleneck machine (machine 3 ) and the succeeding machines (machines 4 and 1). Therefore in this cycle worker 3 does not need to wait for the completion of the operation of worker 3 at machine 2 . Hence the cycle time of worker 2 does not increase in this cycle. These are why the cycle time of worker 2 changes periodically in Figure 2.
On the other hand, when operation times are equal to $S_{1}$, machine 3 is bottleneck for worker 3 , machine 2 for worker 2 and machine 1 for worker 1 . When worker 3 operates at machine 3 , worker 2 tends to operate at machine 2, and when worker 2 operates at machine 2, worker 1 tends to operate at machine 1 . That is, when a certain worker operates at his bottleneck machine, the other workers tend to operate at their bottleneck machines. Therefore, cycle times are the same value in every cycle.

Let us change initial machines of each worker under the condition that the order of arrivals of workers at machine 1 does not change. Worker 1 starts his first operation at machine 1 , worker 2 machine 3 and worker 3 machine 4. Operation times of each worker at each machine is equal to $S_{2}$. Then cycle times of each worker are equal to the results which are shown in Table 2. In all examples which we investigate, initial machines of each worker do not affect cycle times.

Compare sets of operation times $S_{1}$ with $S_{2}$. The set $S_{2}$ is the case that operation times of workers 2 and 3 at each machine are exchanged in the set $S_{1}$. From results of Table 1 and 2, the order of arrivals of workers at machine 1 affects the cycle time.

Figures 3 and 4 are arrow diagrams which show cycle times of worker 1 for successive two cycles. Numbers on vertical axis denote machines and numbers on horizontal axis denote workers. Nodes denote operations of each worker at each machine and numbers on nodes denote operation times of each worker at each machine. Figure 3 shows the case of $S_{1}$ and Figure 4 shows the case of $S_{2}$. Worker 4 is a dummy worker and the operation times are zero. When we follow arrows from the most upper left node, which is referred as to the initial node, to the most upper right node, which is referred as to the last node, if the sum of cycle times on the route is maximal, then the sum is equal to the sum of cycle times of worker 1 for successive two cycles. In Figure 3, on the route that the sum of cycle times is maximal, the sum of cycle times is 16 . This value is equal to the sum of cycle times for successive two cycles in Table 1. Similarly, in Figure 4 the sum is 19 . This value is equals to the sum of cycle times for successive two cycles in Table 2. With Comparing Figures 3 with 4, in Figure 3 there are two nodes at which the operation time is equal to 5 on all routes, while in Figure 4 there are two nodes on which the operation time is equal to 5 on some routes, and there are three nodes on which the operation time is equal to 5 on others.

\section{Conclusions}

In this paper we consider a U-shaped production line with multiple machines and multiple workers under car- 
ousel allocation. In the case that operation times at each machine are different among workers, we investigate the cycle time. Under some specific cases, the cycle time changes periodically. We have some insights into the periodicity of the cycle time and discuss cycle times. For future research the condition that the cycle time changes periodically is derived.

\section{REFERENCES}

[1] Y. Monden, "Toyota production system," An integrated Approach, 3rd edition, Engineering and Management
Press, Georgia, 1998.

[2] G. J. Miltenburg, "One-piece flow manufacturing on u-shaped production lines: A tutorial,” IIE Transactions, Vol. 33, pp. 303-321, 2001.

[3] K. Nakade and K. Ohno, "Separate and carousel type allocations of workers in a U-shaped production line," European Journal of Operational Research, Vol. 145, pp. 403-424, 2003.

[4] K. Nakade and R. Nishiwaki, "Optimal allocation of heterogeneous workers in a U-shaped Production Line," Computers \& Industrial Engineering, Vol. 54, pp. 432440, 2008. 